\title{
Ganglioside Analysis by Thin-Layer Chromatography Matrix-Assisted Laser Desorption/Ionization Orthogonal Time-of-Flight Mass Spectrometry
}

\author{
Vera B. Ivleva* \\ Massachusetts, USA \\ Lisa M. Sapp \\ Perkin-Elmer Life and Analytical Sciences, Boston, Massachusetts, USA
}

Mass Spectrometry Resource, Department of Biochemistry, Boston University School of Medicine, Boston,

Peter B. O'Connor and Catherine E. Costello

Mass Spectrometry Resource, Department of Biochemistry, Boston University School of Medicine, Boston, Massachusetts, USA

\begin{abstract}
Metastable decomposition of ions generated in matrix-assisted laser desorption/ionization (MALDI) mass spectrometers complicates analysis of biological samples that have labile bonds. Recently, several academic laboratories and manufacturers of commercial instruments have designed instruments that introduce a cooling gas into the ion source during the MALDI event and have shown that the resulting vibrational cooling stabilizes these labile bonds. In this study, we compared stabilization and detection of desorbed gangliosides on a commercial orthogonal time-of-flight (oTOF) instrument with results we reported previously that had been obtained on a home-built Fourier transform mass spectrometer. Decoupling of the desorption/ ionization from the detection steps resulted in an opportunity for desorbing thin-layer chromatography (TLC)-separated gangliosides directly from a TLC plate without compromising mass spectral accuracy and resolution of the ganglioside analysis, thus coupling TLC and oTOF mass spectrometry. The application of a declustering potential allowed control of the matrix cluster and matrix adduct formation, and, thus, enhanced the detection of the gangliosides. (J Am Soc Mass Spectrom 2005, 16, 1552-1560) (c) 2005 American Society for Mass Spectrometry
\end{abstract}

$\mathrm{M}$ etastable decomposition occurs universally during matrix-assisted laser desorption/ionization mass spectrometry (MALDI-MS) analyses of molecules having labile bonds. Although this phenomenon can be used for obtaining analyte structural information for small peptides, oligosaccharides, and some glycolipids [1-5] for larger or more fragile molecules, it can limit the mass accuracy and resolution and complicate interpretation of the spectra of heterogeneous samples. Thus, it is important to develop methods that allow control of the extent of metastable decay. In addition to the pressure of the background gas and extraction field strength in the MALDI technique, the major factors affecting metastable fragmen-

Published online July 27, 2005

Address reprint requests to Prof. Catherine E. Costello, Mass Spectrometry Resource, Boston University School of Medicine, 715 Albany Street, R-806, Boston, MA 02118-2526. E-mail: cecmsms@bu.edu

* Also at the Department of Chemistry, Boston University, 590 Commonwealth Avenue, Boston, Massachusetts 02215, USA tation are laser wavelength/pulse width and the choice of the MALDI matrix [6, 7]. In addition, the presence of electrons may play a role in analyte fragmentation and may cause suppression of multiply charged ions in a spectrum $[8,9]$.

Control of the metastable fragmentation in MALDI by increasing the pressure in the ionization source and reduction of the ions internal energy was first introduced by Loboda et al. on orthogonal time-of-flight (oTOF) instruments [10] and later extended to Fourier transform mass spectrometry (FTMS) [11] and to the operation at atmospheric pressure on various types of instruments [12]. These methods have since been used for stabilization of glycolipids $[13,14]$ and peptides and proteins $[11,15]$. The term vibrational cooling (VC) MALDI recently has been introduced to describe desorption in the pressure range where cooling of the excess bond energy is achieved [12]. Stabilization of the labile bonds under these conditions allows for control of the extent of fragmentation, which is particularly 
advantageous for the analysis of heterogeneous samples from biological sources. However, collisional cooling also leads to the stabilization of the matrix adducts and matrix clusters, which may cause analyte signal suppression [16] or otherwise complicate spectral analysis.

Several commercially available and home-built hybrid quadrupole TOF mass spectrometers of the orthogonal design have recently become available. The MALDI Q-Star by MDS/Sciex instrument (Concord, Canada) combines a mass resolving quadrupole and a TOF mass analyzer [17]. Pressure in the MALDI source is maintained at $\sim 11$ mbar to cool the MALDI plume of the gas and to focus an ion beam, but that still does not compensate for the metastable decomposition. Alternatively, an atmospheric pressure MALDI source accommodated on an orthogonal acceleration TOF or an ion trap provides soft ionization, but this technique usually suffers from poor sensitivity compared with conventional MALDI because of a limitation of the ions transmission efficiency [12, 18]. Mid-attomole sensitivity was achieved by using this technique using a countercurrent heated gas stream directed at the sample [19]. Results, obtained on another hybrid MALDI quadrupole-linear ion trap instrument indicated that the optimized operational and instrumental parameters of the atmospheric pressure MALDI source can lead to a substantial increase in sensitivity and overall performance, but compared with the vacuum MALDI, it requires more tuning [20].

Recently, Perkin-Elmer (Boston, MA), in collaboration with MDS/Sciex (Concord, Ontario, Canada), released a new instrument called the prOTOF 2000, which includes a VC-MALDI interface and collisional focusing of the ions into an oTOF detector. Introduction of the collisional cooling source in the prOTOF 2000 instrument allowed for stabilization of the fragile molecules on desorption from the MALDI target [10, 21, 22]. The time the ions spend in the instrument between desorption from the target and detection is typically several milliseconds, which is several orders of magnitude longer than the time of TOF analysis, and, therefore, metastable fragmentation under conditions of low ion source gas pressure is apparent, and stabilization of the ions becomes essential for their detection. The collisional damping interface of the prOTOF 2000 also helps to focus the ions toward the center of the quadrupole ion guide, minimizing the energy spread of the ion beam. The prOTOF typically generates mass accuracy in the $<10-\mathrm{ppm}$ range with resolving power in the $\sim 10,000-15,000$ range. A simple two-point calibration was sufficient for obtaining low parts per million mass accuracy for peptides desorbed from a MALDI target, unlike the multipoint calibration that is required for MALDI-TOF instruments with delayed extraction [23].

The presence of a particularly fragile glycosidic linkage in sialic acid residues, located at the two position, which also bears a free carboxyl group, makes gangliosides the ideal candidates for testing of the

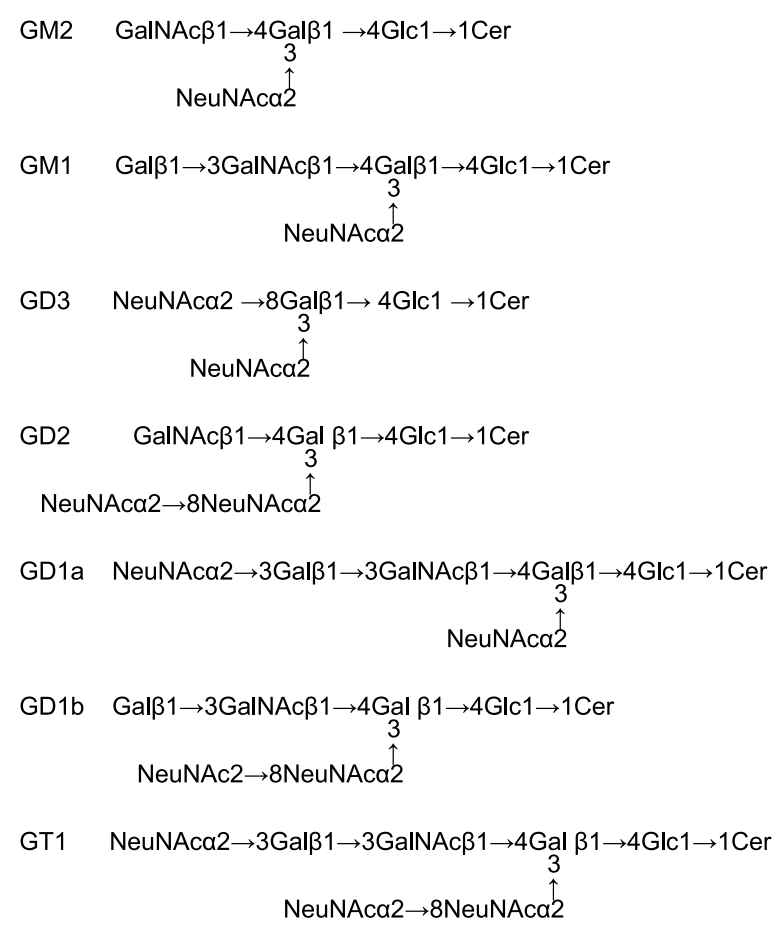

Figure 1. Structures of the gangliosides used in this study (abbreviations correspond to the nomenclature of Svennerholm [38]).

instrument cooling capabilities [11, 14]. Gangliosides belong to a family of complex glycosphingolipids and consist of an oligosaccharide head group containing at least one sialic acid residue, attached to a hydrophobic ceramide anchor (Figure 1). This class of compounds presents a challenge for a mass spectrometric analysis because of its ready elimination of sialic acid $[5,24]$ and the high degree of heterogeneity that frequently characterizes both the carbohydrate and the ceramide portions [25]. Metastable fragmentation of gangliosides results in a loss of structural information and inability to assign the intact species, originally present in the sample. Stabilization of the fragile sialic acid linkages by collisional cooling in MALDI helps to keep them intact and avoids the need for the methyl-esterification procedure that is now routinely applied for analysis of gangliosides in the positive ion mode [26]. Another challenge for characterization of gangliosides is the structural diversity in the carbohydrate moiety and the heterogeneity of both the sphingosine and the longchain base constituents of the ceramide portion of the molecule. The oligosaccharide may contain up to eight sugar units (or occasionally even more); the sialic acid can have a free amine or may be $\mathrm{N}$-acylated or $\mathrm{O}$ acylated and can be present as $\mathrm{N}$-glycoylneuramic acid or $\mathrm{N}$-acetylneuramic acid [27]. The lipid portions can vary in the alkyl chain lengths, level of hydroxylation, saturation, and branching.

Gangliosides are routinely purified and partially characterized by thin-layer chromatography (TLC), followed by extraction of the separated samples from the TLC plate and mass spectrometry analysis. The infor- 
mation obtained from TLC alone should be interpreted with care, because separation of each fraction does not insure its homogeneity and the method itself does not provide high-resolution separation [28, 29]. Any structural variations in the oligosaccharide or lipid moieties, as indicated by spot shifts, should be tested by additional methods. During standard mass spectrometry analysis of TLC-separated spots, a portion of the samples can be lost on extraction out of the silica gel, and the information content of the separation pattern is lost, including evidence for the presence of compounds, other than those toward which a specific reagent was aimed. Direct desorption of the samples from the TLC plate in a MALDI source eliminates these complications and provides simple sample handling combined with the advantages of mass spectrometry methods.

The direct coupling described previously has been implemented on axial MALDI-TOF mass spectrometers, [30-33], showing the feasibility of this method. However, axial MALDI-TOF instruments require a flat surface for sample desorption to achieve optimum resolving power and mass accuracy results. On the other hand, oTOF instruments are not limited in this way. An orthogonal configuration implies decoupling of the desorption and detection steps. This provides an opportunity for the direct desorption of the sample molecules from irregular surfaces such as TLC plates without compromising spectral mass accuracy and resolution. The matrix crystals can have an irregular surface and shape, bringing flexibility in optimizing MALDI preparation technique for the TLC procedure.

The goal of this study was to evaluate the performance of the prOTOF 2000 instrument with respect to its cooling capabilities and the possibility of its direct coupling with the TLC method. The following data show that cleavage of the sialic acid linkages can be minimized by collisional cooling, followed by an individual ganglioside assignment for the set of the ganglioside standards and the whole brain ganglioside mix. Previous experience [14] shows that such coupling requires a higher quantity of matrix, which often results in matrix adducts and cluster peaks. The declustering voltage provides a low level of excitation, and this was explored as a potential tool for minimizing contributions from such ions to the spectra while testing whether the stability of the gangliosides can be affected by this mild excitation.

\section{Experimental}

The gangliosides GM1, GM2, GD1a, GD1b, and GT1b and whole brain ganglioside mix were purchased from Sigma-Aldrich (St. Louis, MO); GM3 and GD2 were purchased from Fisher Scientific (Pittsburgh, PA). Silica gel on aluminum TLC plates obtained from SigmaAldrich was used. The optimized TLC separation procedure [14] was applied, using a solution of sinapinic acid matrix (Fluka, Buchs, Switzerland) in methanol/ acetonitrile/water (2:2:1), and spotting on top of the
TLC plate after the separation. The concentration of the matrix $(50 \mu \mathrm{g} / \mathrm{mL})$ was slightly decreased compared with the previously developed methods, where highly saturated (up to $100 \mu \mathrm{g} / \mathrm{mL}$ ) solutions were used. The two TLC plates were developed under identical conditions; one plate was sprayed with a detecting reagent and the preliminary position of the spots on the second plate was determined, followed by laser scanning in the mass spectrometer. For the analysis of biological mixtures available only in small amounts, the standard ganglioside set alone was applied onto a reference TLC plate to spare the precious sample. The MALDI target was placed on a computer-controlled $X, Y$ stage [23]. A nitrogen laser (337ND-Sx; SpectraPhysics, Mountain View, CA) generated 500 laser shots at $20 \mathrm{~Hz}$; at this rate each acquisition was accomplished in $26 \mathrm{~s}$. The full length of each TLC strip was scanned in $0.5-\mathrm{mm}$ increments, whether there were spots detected by a visualizing reagent or not, because of the possible presence of low abundance components that could have been missed by chemical visualization, because previous results had indicated that the mass spectrometer is likely to be more sensitive than the chemical visualization methods.

All experiments were performed on an orthogonal MALDI-TOF (PrOTOF) instrument. Pulsing $\mathrm{N}_{2}$ gas during the desorption/ionization event provided the maximum cooling flow and a focusing flow of $212 \mathrm{~mL} / \mathrm{min}$, which corresponds to the pressure of $\sim 1 \mathrm{mbar}$ at the MALDI target. The cooling flow, along with the laser fluence, was optimized to achieve minimum ganglioside fragmentation, while the focusing flow was held constant at $210 \mathrm{~mL} / \mathrm{min}$. The declustering potential between the cone and the MALDI target was varied within the range of $5-60 \mathrm{~V}$, while the cone voltage was held constant at $-38 \mathrm{~V}$. The other ion optic voltages were set as follows in the negative ion mode: $-31 \mathrm{~V}$ at quadrupole offset, $-29 \mathrm{~V}$ at the orifice, and $-28.5 \mathrm{~V}$ at the mesh. "Push" and "pull" voltages were set at -1465 and $1465 \mathrm{~V}$, respectively, and the accelerating voltage was set to $16 \mathrm{kV}$. The detector was biased at $2.3 \mathrm{kV}$, the reflectron plate in the dual stage reflector was set at $-2.2 \mathrm{kV}$, and the reflectron grid was set at $1460 \mathrm{~V}$. A two-point external calibration was performed using PepMix 1 from LaserBioLabs (Sophia, France) and yielded a calibration curve that was accurate to $\sim 5 \mathrm{ppm}$ for abundant peaks.

\section{Results and Discussion}

Although gangliosides are routinely analyzed in the negative ion mode, the preliminary cooling data were obtained in the positive ion mode, where the asialopeaks were easily observed, allowing observation of the full fragmentation pattern [14]. In these experiments, the spectra of even the standard gangliosides, desorbed from a glass-coated gold plate, generally contain traces of fragment products. Because the observed de-sialylated species could be attributed to either the metastable 


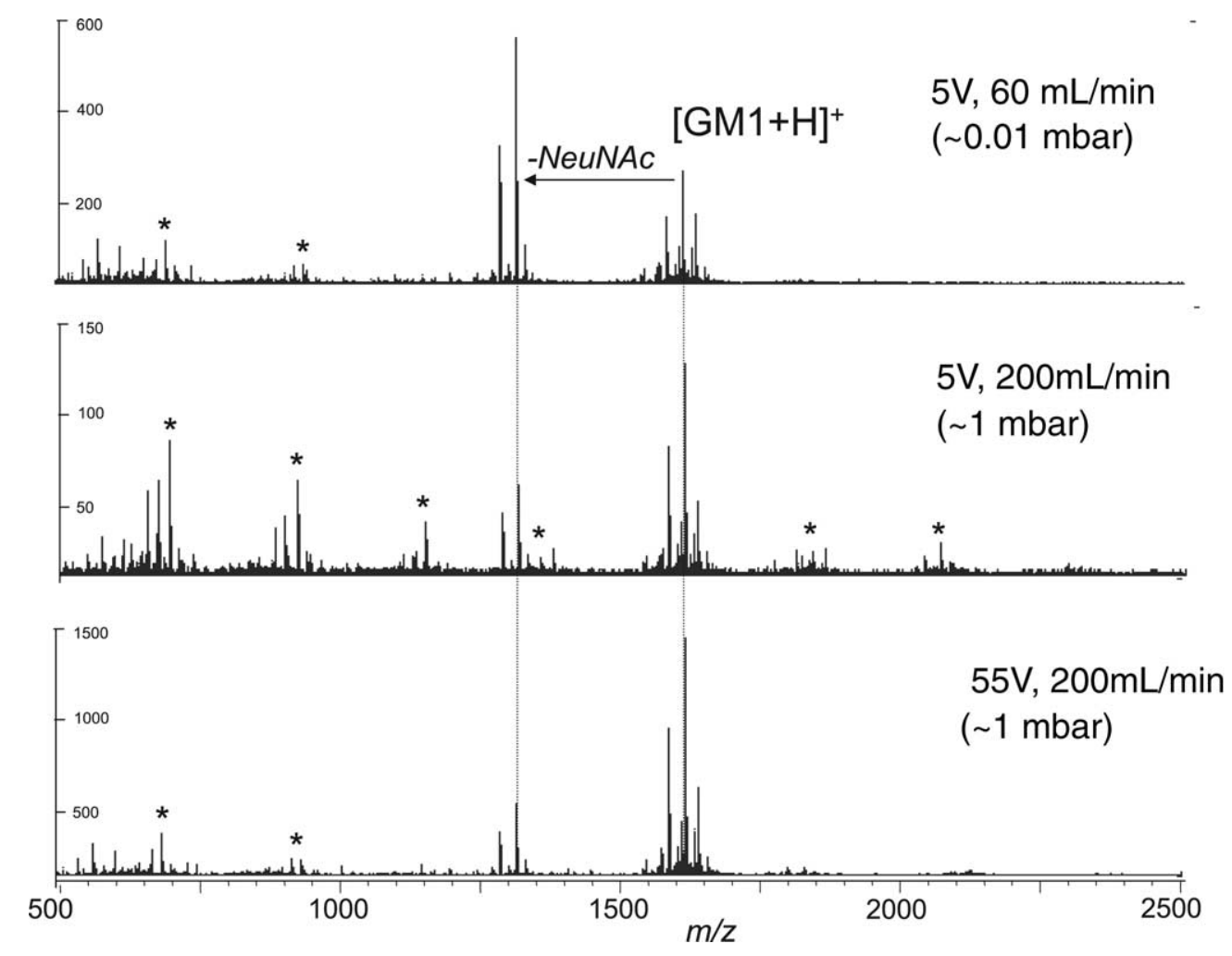

Figure 2. Effect of the cooling flow and declustering potential on the ganglioside stabilization in the positive ion mode. Asterisks denote matrix adducts and clusters.

fragmentation in the source or the actual solution content, the goal of this optimization step was to minimize the ion fragmentation.

Under conditions of a 5-V declustering voltage and low cooling flow rates, corresponding to pressure of $0.01 \mathrm{mbar}$ at the MALDI target, the ganglioside fragmentation was apparent (Figure 2a). The major peak observed was asialo-GM1, and the abundance of the ions indicative of GM1 molecular weight was relatively low. Increasing the cooling gas flow up to $200 \mathrm{~mL} / \mathrm{min}$ raised the ion source pressure up to $\sim 1 \mathrm{mbar}$, and significant cooling was achieved, as shown by the efficient stabilization of the ganglioside molecule (Figure $2 b$ ). As a consequence of the stabilization effect, the matrix clusters and adducts became abundant, complicating interpretation of the spectra and suppressing the analyte signal. Increasing the declustering voltage to 55 $\mathrm{V}$ allowed for a gentle excitation and resulted in dissociation of the matrix complexes without compromising the stability of the gangliosides themselves; this led to significant reduction of the chemical noise and higher signal intensity (Figure 2c). The laser fluence was adjusted manually to obtain a reliable signal without inducing ganglioside fragmentation.

This approach of varying the declustering voltage has been investigated and successfully applied for removing matrix adducts during the analysis of the proteins, [23] and the mechanism of the adduct formation has been investigated [16]. As shown by Krutchinsky et al., [34] activation energies of the matrix clusters are lower than those used to fragment peptide ions. Here, we show that even such fragile molecules as gangliosides could be dissociated from unwanted adducts by gentle excitation, while still keeping labile linkages intact. Increase of the analyte peak intensity after declustering voltage application illustrated an adverse effect of the chemical noise on signal detection and supported the idea that matrix clusters and adducts are significant MALDI contaminants [34]. Because desorption off the TLC surface required a significant amount of matrix spotted on a plate, as shown later, application of the declustering voltage becomes necessary to reduce the presence of abundant matrix clusters and adducts.

The optimized conditions from Figure 2 were applied for the analysis of the gangliosides desorbed from the gold-coated glass MALDI plate (Figure 3). Minimization of the sialic acid loss was achieved, and each peak could be unambiguously assigned to an individual ganglioside species. The water loss observed in the GD1b spectra (Figure 3d) was probably the result of lactone formation between two $\alpha 2,8$-linked sialic acid residues, which takes place in solution under acidic conditions $[35,36]$. Although they have the same mass, the two GD1 isomers can be distinguished on the basis of differences in the relative abundance of their fragment ions [25], most clearly in the positive ion mode, as shown by the spectra in Figure 4. GD1a and GD1b have two sialic acid residues. These are attached by $\alpha 2,3-$ linkages to different galactose residues along the GD1a 


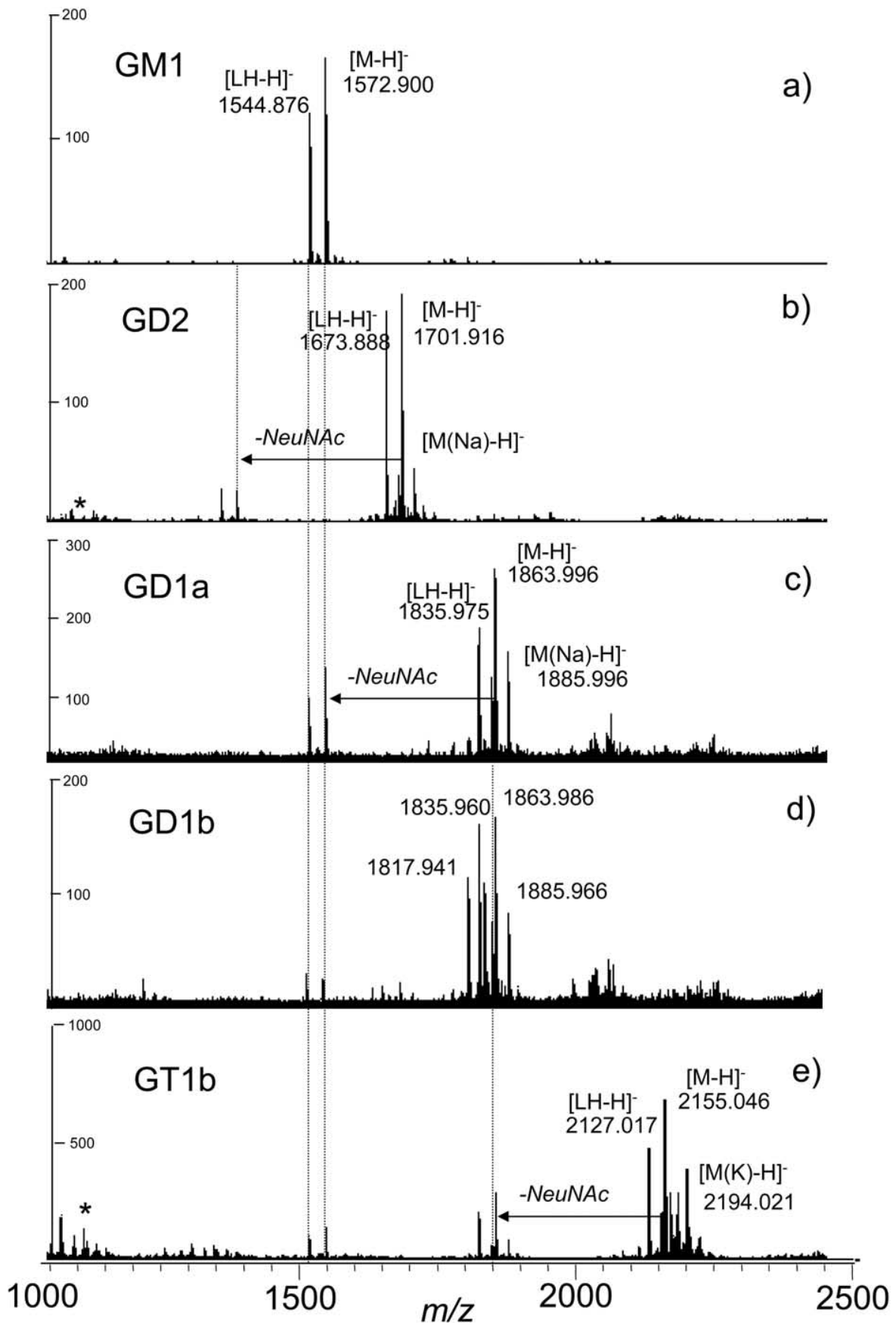

Figure 3. Negative ion mode MALDI mass spectra of the gangliosides, desorbed with collisional cooling, from a glass/gold MALDI plate: (a) GM1, (b) GD2, (c) GD1a, (d) GD1b, and (e) GT1b.

oligosaccharide head group, whereas in GD1b an $\alpha 2,8-$ linked-disialyl moiety is $\alpha 2,3$-linked to the interior galactose residue (Figure 1). Because the sialylated residues are attached to equivalent positions in GD1a, the probability of sialic acid loss from either position is roughly equal, and this results in the relatively high abundance of the signals corresponding to the $[\mathrm{M}+$ $\mathrm{Na}^{+}$monosialic species $(\mathrm{m} / \mathrm{z} 1597$ for the higher homolog and $m / z 1569$ for the lower homolog). In contrast, the GD1b fragmentation pattern indicates that the $\alpha 2,8-$ glycosidic bond between the two sialic acid residues is stronger than the $\alpha 2,3$-linkage between the inner sialic acid and the backbone galactose. Therefore, when fragmentation occurs, the asialo-fragment is substantially more abundant than the mono-sialo-fragment in the positive ion mode MALDI mass spectra of GD1b $(\mathrm{m} / \mathrm{z}$ 1306 for the higher homolog and $\mathrm{m} / \mathrm{z} 1278$ for the lower homolog) [14, 26]. This characteristic pattern can be helpful for distinction between the isomers.

The next set of experiments was performed with the standard gangliosides and whole brain extract mixes applied and separated on a TLC plate. The conditions 


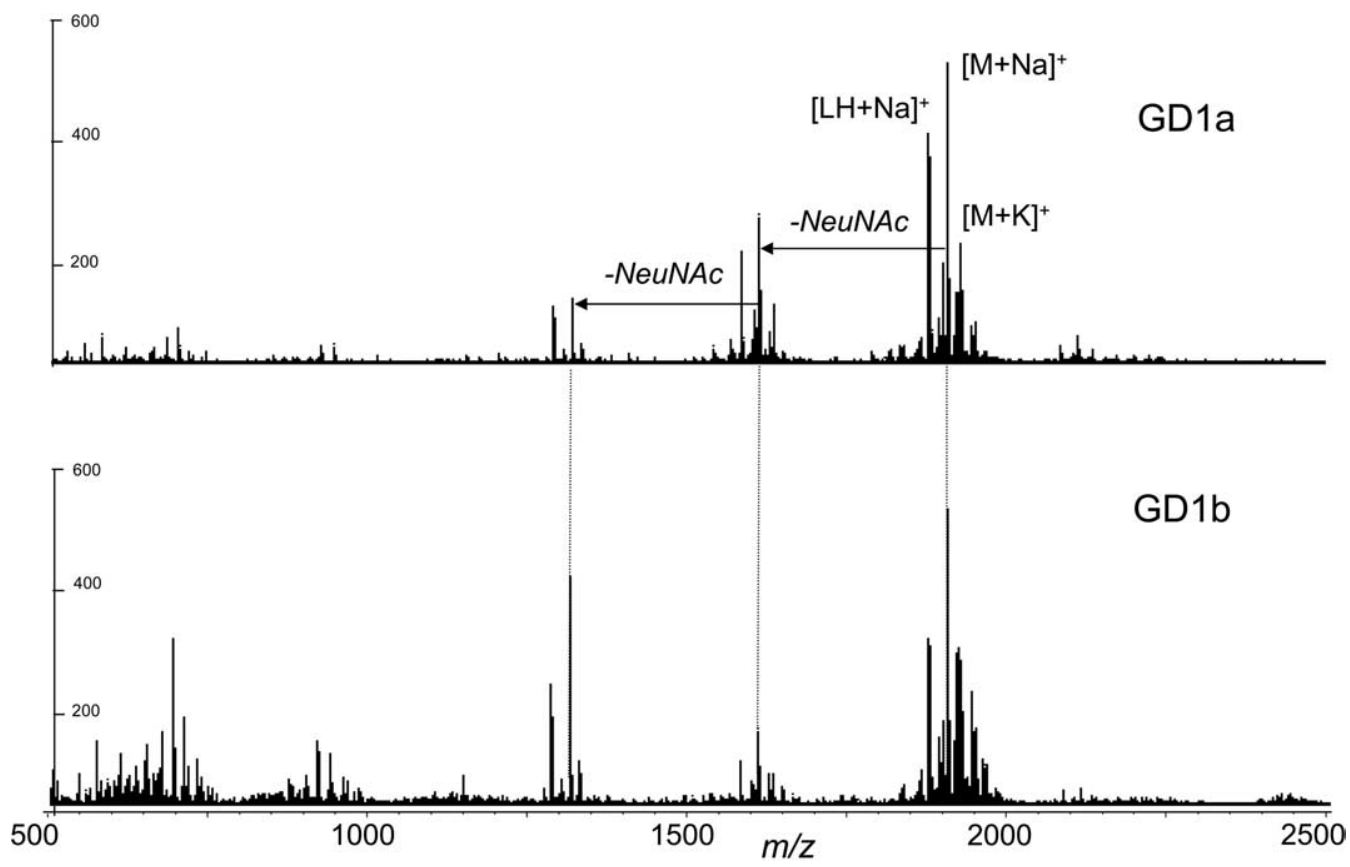

Figure 4. Differentiation of the GD1 isomers desorbed from the TLC plate in the positive ion mode, $55-\mathrm{V}$ declustering voltage, $200 \mathrm{~mL} / \mathrm{min}$.

for desorbing the gangliosides off of the TLC plates were optimized, including the type and amount of matrix required for the best spectral performance. Sinapinic acid was the best matrix for this purpose, because it provides the most homogeneous cocrystallization within the bulk silica gel layer on the TLC plate and cocrystallizes with the gangliosides most efficiently, as was reported previously [14]. For VC-MALDI-FTMS, a



Figure 5. Molecular ion region in the negative ion mode mass spectra of the standard gangliosides: (a) GM2, (b) GD3 and whole brain gangliosides, (c) GM1, (d) GD1a, and (e) GT1b, obtained by direct coupling of TLC with prOTOF using sinapinic acid as the matrix. 
concentrated solution of the matrix $(50 \mu \mathrm{g} / \mathrm{mL})$ was required for desorbing the samples off the TLC plate; whereas a lower matrix concentration $(10-20 \mu \mathrm{g} / \mathrm{mL})$ was best for desorbing samples off of the metal target. Highly saturated matrix solutions (up to $100 \mu \mathrm{g} / \mathrm{mL}$ ) were tried at first, but these resulted in abundant matrix clusters and adducts and suppression of the ganglioside signal. Therefore, for these MALDI-oTOF-MS analyses, the matrix concentration was reduced compared with the method that had been optimized for VC-FTMS. A highly volatile solvent was used for the matrix to promote fast crystal formation and avoid sample spreading. The positions of the ganglioside standards on the TLC plate were found by comparing matching positions of the spots on the developed TLC plate, stained with a specific reagent. The lane containing the mix of whole brain ganglioside lanes was scanned with the laser along its entire length, in $0.5-\mathrm{mm}$ increments, to probe for species other than the standards. One of the lanes with the whole brain ganglioside mix was set too close to the TLC plate right edge (Figure 5) and the separated compounds exhibited increased $R_{\mathrm{f}}$ values, a result that could be misleading for assignment of the individual species, thereby demonstrating one of the disadvantages of the planar separation method. However, mass spectroscopy scanning enabled accurate identification of all spots in that lane.

To evaluate mass accuracy under these conditions, a series of parallel measurements of the molecular masses of the ganglioside standards was performed for samples desorbed from a glass-coated gold plate and the same samples desorbed from a TLC plate. For the samples desorbed from the relatively flat metal plate, a root mean square (rms) error of $4.1 \mathrm{ppm}$, with standard deviation of $3.7 \mathrm{ppm}$, was obtained by an external calibration, whereas an rms error of $6.2 \mathrm{ppm}$ and standard deviation of $6.2 \mathrm{ppm}$ were obtained for the samples desorbed from the porous surface of a TLC plate (Tables 1 and 2). Spotting a large amount of matrix on top of a developed TLC plate resulted in a slight bulging of the TLC surface in the area of the analyte spot, and the matrix crystals, formed from a highly saturated solution, were very prominent. Neither the uneven surface of the TLC plate nor the jagged surface of the matrix crystals affected the spectral mass accuracy or resolution. This outcome was expected, based on the decoupling of the desorption/ionization from detection steps because of the orthogonal configuration of the instrument.

The sensitivity of the method allowed for measuring a high-quality spectrum when 10 pmol of the ganglioside sample was spotted on the TLC plate before separation. Although this sample requirement is not impressive for modern mass spectrometers, it is important to note that the signal is proportional to the number of ions desorbed from the area illuminated by the laser $(200 \times 230 \mu \mathrm{m})$, and the diameter of the developed analyte spot is $\sim 5 \times 3 \mathrm{~mm}$, and the spot diffusion
Table 1. Mass accuracy of measurements for gangliosides desorbed from glass-gold MALDI plate

\begin{tabular}{|c|c|c|c|}
\hline Ganglioside & $\begin{array}{l}\text { Predicted } \\
m / z(\mathrm{M}-\mathrm{H})-\end{array}$ & $\begin{array}{c}\text { Observed } \\
\mathrm{m} / \mathrm{z}\end{array}$ & $\begin{array}{l}\text { Error } \\
\text { (ppm) }\end{array}$ \\
\hline \multirow[t]{3}{*}{ GM1 } & 1572.9007 & 1572.9057 & 3.2 \\
\hline & & 1572.8977 & -1.9 \\
\hline & & 1572.9039 & 2.0 \\
\hline \multirow[t]{3}{*}{ GM1 (LH) } & 1544.8694 & 1544.8749 & 3.6 \\
\hline & & 1544.8511 & -11.8 \\
\hline & & 1544.8734 & 2.3 \\
\hline \multirow[t]{3}{*}{ GM2 } & 1410.8479 & 1410.8445 & -2.4 \\
\hline & & 1410.8438 & -2.9 \\
\hline & & 1410.8404 & -5.3 \\
\hline \multirow[t]{3}{*}{ GM2 (LH) } & 1382.8166 & 1382.8153 & -0.9 \\
\hline & & 1382.8140 & -1.9 \\
\hline & & 1382.8134 & -2.3 \\
\hline \multirow[t]{3}{*}{ GD1a } & 1863.9961 & 1863.9988 & 1.4 \\
\hline & & 1863.9997 & 1.9 \\
\hline & & 1863.9838 & -6.5 \\
\hline \multirow[t]{3}{*}{ GD1a (LH) } & 1835.9648 & 1835.9635 & -0.7 \\
\hline & & 1836.9729 & 4.4 \\
\hline & & 1835.9625 & -1.3 \\
\hline \multirow[t]{3}{*}{ GD1b } & 1863.9961 & 1863.9893 & -3.6 \\
\hline & & 1863.9864 & -5.2 \\
\hline & & 1863.9942 & -1.0 \\
\hline \multirow{3}{*}{ GD1b (LH) } & 1835.9658 & 1835.9683 & 1.9 \\
\hline & & 1835.9599 & -2.6 \\
\hline & & 1835.9570 & -4.2 \\
\hline \multirow[t]{3}{*}{ GD3 (LH) } & 1470.8326 & 1470.8187 & -9.5 \\
\hline & & 1470.8263 & -4.2 \\
\hline & & 1470.8277 & -3.3 \\
\hline \multirow[t]{3}{*}{ GT1b } & 2155.0915 & 2155.0923 & 0.4 \\
\hline & & 2155.0852 & -2.9 \\
\hline & & 2155.0870 & -2.1 \\
\hline
\end{tabular}

$\mathrm{rms}(\mathrm{ppm})=4.1 ;$ standard deviation $(\mathrm{ppm})=3.7$.

extends to the depth of $0.5 \mathrm{~mm}$ within the silica coating. The lateral spread of the analyte and its penetration into the silica surface during the spot migration contributed to the apparent "low" sensitivity. Because of its deeper penetration into the surface, an infrared laser (Er:YAG, $2.94 \mu \mathrm{M})$ has shown a sensitivity increase, compared with UV-MALDI, for desorption of glycolipid ions from a TLC plate [30], but the instrument available at that time (1998) did not have orthogonal injection, and the massive resulting plume degraded the spectral quality. One must keep in mind, however, that for a typical TLC experiment that uses color reagents for detection, the samples often are spotted in the high picomole-nanomole range. Thus, although the presently achieved 10-pmol sensitivity may seem low when compared with other types of mass spectrometry analyses, direct UVMALDI-MS is still sufficient for many TLC experiments. Compared with the usual TLC detection methods, it offers a substantial increase in both sensitivity and specificity. Furthermore, continuous improvements in the methodology described here and the alternative use of an infrared laser for desorption can be expected to lower the detection limits. Experiments of the latter type are underway elsewhere [39]. 
Table 2. Mass accuracy of measurements for gangliosides desorbed directly from the TLC plate

\begin{tabular}{|c|c|c|c|}
\hline Ganglioside & $\begin{array}{l}\text { Predicted } \\
m / z(\mathrm{M}-\mathrm{H})-\end{array}$ & $\begin{array}{c}\text { Observed } \\
\mathrm{m} / \mathrm{z}\end{array}$ & $\begin{array}{r}\text { Error } \\
\text { (ppm) }\end{array}$ \\
\hline \multirow[t]{3}{*}{ GM1 } & 1572.9007 & 1572.8861 & -9.3 \\
\hline & & 1572.9220 & 13.5 \\
\hline & & 1572.9147 & 8.9 \\
\hline \multirow[t]{3}{*}{ GM1 (LH) } & 1544.8694 & 1544.8672 & 1.5 \\
\hline & & 1544.8687 & -0.5 \\
\hline & & 1544.8766 & 4.7 \\
\hline \multirow[t]{3}{*}{ GM2 } & 1410.8479 & 1410.8388 & -6.4 \\
\hline & & 1410.8342 & -9.7 \\
\hline & & 1410.8468 & -0.7 \\
\hline \multirow[t]{3}{*}{ GM2 (LH) } & 1382.8166 & 1382.8026 & -10.1 \\
\hline & & 1382.8096 & -5.1 \\
\hline & & 1382.8220 & 3.9 \\
\hline \multirow[t]{3}{*}{ GD1a } & 1863.9961 & 1863.9884 & -4.1 \\
\hline & & 1863.9873 & -4.7 \\
\hline & & 1863.9879 & -4.4 \\
\hline \multirow[t]{3}{*}{ GD1a (LH) } & 1835.9648 & 1835.9674 & 1.4 \\
\hline & & 1836.9590 & -3.2 \\
\hline & & 1835.9642 & -5.8 \\
\hline \multirow[t]{3}{*}{ GD1b } & 1863.9961 & 1863.9953 & -0.4 \\
\hline & & 1863.9918 & -2.3 \\
\hline & & 1863.9893 & -3.6 \\
\hline \multirow[t]{3}{*}{ GD1b (LH) } & 1835.9658 & 1835.9491 & 8.6 \\
\hline & & 1835.9615 & 1.8 \\
\hline & & 1835.9612 & -2.0 \\
\hline \multirow[t]{3}{*}{ GD3 (LH) } & 1470.8326 & 1470.8324 & -0.1 \\
\hline & & 1470.8524 & 13.5 \\
\hline & & 1470.8282 & -3.0 \\
\hline \multirow{3}{*}{ GT1b } & 2155.0915 & 2155.1060 & 6.7 \\
\hline & & 2155.0867 & -2.2 \\
\hline & & 2155.0771 & -6.7 \\
\hline
\end{tabular}

$\mathrm{rms}(\mathrm{ppm})=6.2 ;$ standard deviation $(\mathrm{ppm})=6.2$.

\section{Conclusions}

Coupling of the TLC method with the prOTOF 2000 instrument yielded a simple and fast analysis for gangliosides. Vibrational stabilization of the fragile molecular ions generated from the gangliosides was achieved by using the collisional cooling ion source and resulted in an unambiguous assignment of the individual species from each TLC spot. The orthogonal configuration of the prOTOF instrument allowed for direct desorption of samples from the irregular surface of the TLC plate without impairment of either mass accuracy or resolution.

The unwanted matrix clusters and matrix adducts readily formed in the collisionally cooled source were dissociated by application of a declustering voltage; yet, the sialic acid linkages were kept intact. The amount of matrix adducts and clusters in the gas phase is proportional to the MALDI plume density, and, therefore, chemical noise of this origin can be reduced by preparing a less dense matrix crystal packing [16]. This approach could lead to a decrease in the sensitivity of the method, because, along with the matrix, a lower amount of the analyte would be desorbed from such MALDI spots. For this reason, considerable attention needs to be given to the search for the proper matrixto-analyte ratio, to obtain clean spectra with minimum matrix adduction. On the other hand, the prOTOF design supplies a constant cooling rate in the ionization source, whereas the FTMS instrument with the VC source [37] allows variation of the timing of the cooling gas pulse, with respect to the ionization/desorption event. Coordination of these two events may help reduce the amount of matrix adducts, if declustering voltage is applied immediately after the ionization, rather than simultaneously with it.

The flight time of ions through the TOF tube from the ion source to the detector is typically $\sim 1-10 \mathrm{~ms}$, but the laser user has a maximum pulse rate of $20 \mathrm{~Hz}$. Thus, replacement of the laser with one that pulses in the 0.1to $1-\mathrm{kHz}$ range would clearly improve the high throughput parameter of the instrument. This will be highly advantageous to users who are interested in scanning entire two-dimensional TLC plates. Furthermore, the same instrument can be used, with little or no modification, for scanning of other surfaces, such as SPR (surface plasmon resonance), polyacrylamide gel electrophoresis, or antibody arrays.

\section{Acknowledgments}

This project was funded in part with Federal funds from the National Institutes of Health, under grant no. P41 RR10888 from the National Center for Research Resources and under a research collaboration agreement between Perkin-Elmer and the Boston University School of Medicine (BUSM).

\section{References}

1. Spengler, B.; Kirsch, D.; Kaufmann, R. Metastable Decay of Peptides and Proteins in Matrix-Assisted Laser-Desorption Mass-Spectrometry. Rapid Commun. Mass Spectrom. 1991, 5, 198-202.

2. Brown, R. S.; Lennon, J. J. Sequence-Specific Fragmentation of Matrix-Assisted Laser-Desorbed Protein Peptide Ions. Anal. Chem. 1995, 67, 3990-3999.

3. Ngoka, L. C.; Gal, J. -F.; Lebrilla, C. B. Effects of Cations and Charge Types on the Metastable Decay Rates of Oligosaccharides. Anal. Chem. 1994, 66, 692-698.

4. Viseux, N.; Costello, C. E.; Domon, B. Post-Source Decay Mass Spectrometry: Optimized Calibration Procedure and Structural Characterization of Permethylated Oligosaccharides. J. Mass Spectrom. 1999, 34, 364-376.

5. Costello, C. E.; Juhasz, P.; Perreault, H. New Mass Spectral Approaches to Ganglioside Structure Determinations. Biol. Brain Res. 1994, 101, 45-61.

6. Karas, M.; Bahr, U.; Strupat, K.; Hillenkamp, F. Matrix Dependence of Metastable Fragmentation of Glycoproteins in MALDI TOF Mass Spectrometry. Anal. Chem. 1995, 67, 675679.

7. Berkenkamp, S.; Menzel, C.; Karas, M.; Hillenkamp, F. Performance of Infrared Matrix-Assisted Laser Desorption/Ionization Mass Spectrometry with Lasers Emitting in the $3 \mu \mathrm{M}$ Wavelength Range. Rapid Commun. Mass Spectrom. 1997, 11, 1399-1406.

8. Karas, M.; Gluckmann, M.; Schafer, J. Ionization in MatrixAssisted Laser Desorption/Ionization: Singly Charged Molecules Are the Lucky Survivors. J. Mass Spectrom. 2000, 35, 1-12. 
9. Frankevich, V.; Zhang, J.; Dashtiev, M.; Zenobi, R. Production and Fragmentation of Multiply Charged Ions in "ElectronFree" Matrix-Assisted Laser Desorption/Ionization. Rapid Commun. Mass Spectrom. 2003, 17, 2343-2348.

10. Krutchinsky, A. N.; Loboda, A. V.; Spicer, V. L.; Dworschak, R.; Ens, W.; Standing, K. G. Orthogonal Injection of MatrixAssisted Laser Desorption/Ionization Ions into a Time-ofFlight Spectrometer through a Collisional Damping Interface. Rapid Commun. Mass Spectrom. 1998, 12, 508-518.

11. O'Connor, P. B.; Costello, C. E. A High Pressure MatrixAssisted Laser Desorption/Ionization Fourier Transfrom Mass Spectrometry Ion Source for Thermal Stabilization of Labile Biomolecules. Rapid Commun. Mass Spectrom. 2001, 15, 1862-1868.

12. Laiko, V. V.; Baldwin, M. A.; Burlingame, A. L. Atmospheric Pressure Matrix Assisted Laser Desorption/Ionization Mass Spectrometry. Anal. Chem. 2000, 72, 652-657.

13. O'Connor, P. B.; Mirgorodskaya, E.; Costello, C. E. High Pressure Matrix-Assisted Laser Desorption/Ionization Fourier Transform Mass Spectrometry for Minimization of Ganglioside Fragmentation. J. Am. Soc. Mass Spectrom. 2002, 13, 402-407.

14. Ivleva, V. B.; Elkin, Y. N.; Budnik, B. A.; Moyer, S. C.; O'Connor, P. B.; Costello, C. E. Coupling Thin Layer Chromatography with Vibrational Cooling Matrix-Assisted Laser Desorption/Ionization Fourier Transform Mass Spectrometry for the Analysis of Ganglioside Mixtures. Anal. Chem. 2004, 76, 6484-6512.

15. Moyer, S. C.; Budnik, B. A.; Pittman, J. L.; Costello, C. E.; O'Connor, P. B. Attomole Peptide Analysis by High-Pressure Matrix-Assisted Laser Desorption/Ionization Fourier Transform Mass Spectrometry. Anal. Chem. 2003, 75, 6449-6454.

16. Loboda, A. V.; Chernushevich, I. V. Investigation of the Mechanism of Matrix Adduct Formation in Maldi at Elevated Pressure. Int. J. Mass Spectrom. 2005, 240, 101-105.

17. Baldwin, M. A.; Medzihradszky, K. F.; Lock, C. M.; Fisher, B.; Settineri, T. A.; Burlingame, A. L. Matrix Assisted Laser Desorption/Ionization Coupled with Quadrupole/Orthogonal Acceleration Time-of-Flight Mass Spectrometry for Protein Discovery, Identification, and Structural Analysis. Anal. Chem. 2001, 73, 1707-1720.

18. Laiko, V. V.; Moyer, S. C.; Cotter, R.J. Atmospheric Pressure MALDI/Ion Trap Mass Spectrometry. Anal. Chem. 2000, 72, 5239-5243.

19. Miller, C. A.; Yi, D. H.; Perkins, P. D. An Atmospheric Pressure Matrix-Assisted Laser Desorption/Ionization Ion Trap with Enhanced Sensitivity. Rapid Commun. Mass Spectrom. 2003, 17, 860-868.

20. Schneider, B. B.; Lock, C.; Covey, T. R. AP and Vacuum MALDI on a QqLIT Instrument. J. Am. Soc. Mass Spectrom. 2005, 16, 176-182.

21. Guilhaus, M.; Selby, D.; Mlynski, V. Orthogonal Acceleration Time-of-Flight Mass Spectrometry. Mass Spectrom. Rev. 2000, 19, 65-107.

22. Chernushevich, I. V.; Loboda, A. V.; Thomson, B. A. An Intoduction to Quadrupole-Time-of-Flight Mass Spectrometry. J. Mass Spectrom. 2001, 36, 849-865.

23. Loboda, A. V.; Ackloo, S.; Chernushevich, I. V. A HighPerformance Matrix-Assisted Laser Desorption/Ionization Orthogonal Time-of-Flight Mass Spectrometer with Colli- sional Cooling. Rapid Commun. Mass Spectrom. 2003, 17 $2508-2516$.

24. Harvey, D. J. Matrix-Assisted Laser Desorption/Ionization Mass Spectrometry of Carbohydrates. Mass Spectrom. Rev. 1999, 18, 349-451.

25. Wiegandt, $\mathrm{H}$. The Chemical Composition of Gangliosides of the Vertebrate Nervous System. Behav. Brain Res. 1995, 66, 85-97.

26. Juhasz, P.; Costello, C. E. Matrix-Assisted Laser Desorption Ionization Time-of-Flight Mass Spectrometry of Underivatized and Permethylated Gangliosides. J. Am. Soc. Mass Spectrom. 1992, 3, 785-796.

27. Schauer, R. Sialic Acids: Chemistry, Metabolism, and Function; Springer-Verlag: New York, 1982.

28. Kanfer, J. N.; Hakomori, S. Sphingolipid Biochemistry; New York: Plenum Press, 1983.

29. Hakomori, S.; Nudelman, E.; Levery, S. B.; Kannagi, R. Novel Fucolipids Accumulating in Human Adenocarcinoma. I. Glycolipids with Di- or Trifucosylated Type 2 Chain. J. Biol. Chem. 1984, 259, 4672-4680.

30. Guittard, J.; Hronowski, X. L.; Costello, C. E. Direct MatrixAssisted Laser Desorption/Ionization Mass Spectrometric Analysis of Glycosphingolipids on Thin Layer Chromatographic Plates and Transfer Membranes. Rapid Commun. Mass Spectrom. 1999, 13, 1838-1849.

31. Gusev, A. I.; Vasseur, O. J.; Proctor, A.; Sharkey, A. G.; Hercules, D. M. Imaging of Thin-Layer Chromatograms Using Matrix-Assisted Laser Desorption/Ionization Mass Spectrometry. Anal. Chem. 1995, 67, 4565-4570.

32. Mehl, J. M.; Hercules, D. M. Direct TLC-MALDI Coupling Using a Hybrid Plate. Anal. Chem. 2000, 72, 68-73.

33. Crecelius, A.; Clench, M. R.; Richards, D. S.; Parr, V. ThinLayer Chromatography-Matrix-Assisted Laser Desorption Ionization-Time-of-Flight Mass Spectrometry Using Particle Suspension Matrices. J. Chromatogr. A 2002, 958, 249-260.

34. Krutchinsky, A. N.; Chait, B. T. On the Nature of the Chemical Noise in MALDI Mass Spectra. J. Am. Soc. Mass Spectrom. 2002, 13, 129-134.

35. Bassi, R.; Riboni, L.; Sonnino, S.; Tettamanti, G. Lactonization of GD1b Ganglioside under Acidic Conditions. Carbohydr. Res. 1989, 193, 141-146.

36. Perreault, H.; Costello, C. E. Stereochemical Effects on the Mass Spectrometric Behavior of Native and Derivatized Trisaccharide Isomers: Comparisons with Results from Molecular Modeling. J. Mass Spectrom. 1999, 34, 184-197.

37. O'Connor, P. B.; Budnik, B. A.; Ivleva, V. B.; Kaur, P.; Moyer, S. C.; Pittman, J. L.; Costello, C. E. A High Pressure MatrixAssisted Laser Desorption Ion Source for Fourier Transform Mass Spectrometry Designed to Accommodate Large Targets With Diverse Surfaces. J. Am. Soc. Mass Spectrom. 2003, 15, 128-132.

38. Svennerholm, L. Chromatographic Separation of Human Brain Gangliosides. J. Neurochem. 1963, 10, 613-623.

39. Dreisewerd, K., Müthing, J., Rohlfing, A., Meisen, I., Vukelić, Ž., Peter-Katalinić, J., Hillenkamp, F., Berkenkamp, S. Analysis of Gangliosides Directly from Thin-layer Chromatography Plates by Infrared Matrix-Assisted Laser Desorption/Ionization Orthogonal Time-of-Flight Mass Spectrometry with a Glycerol Matrix. Anal. Chem. 2005, in press. 